\title{
Expression and regulation of phenol-soluble modulins and enterotoxins in foodborne Staphylococcus aureus
}

\author{
Xiaoxiao $\mathrm{Wu}^{1,2+}{ }^{+}$, Miao Yang ${ }^{1 \dagger}$, Xin Fang ${ }^{1}$, Shiqi Zhen ${ }^{3}$, Jie Zhang ${ }^{4}$, Xiangying Yang ${ }^{4}$, Ling Qiao ${ }^{1}$, Yang Yang ${ }^{1}$ \\ and Chi Zhang ${ }^{1 *}$
}

\begin{abstract}
Although high levels of staphylococcal phenol-soluble modulins (PSMs) in clinical methicillin-resistant Staphylococcus aureus (MRSA) has been shown to correlate with bacterial virulence, the PSMs expression in foodborne Staphylococcus aureus (S. aureus), as well as its association with staphylococcal food poisoning (SFP) was not yet clear. We collected a panel of 350 foodborne and 127 clinic-derived S. aureus strains and compared their PSMs expression. Overall, foodborne strains exhibited higher PSMs than clinical isolates, indicating a potential pathological significance of PSMs in staphylococcal food contamination. Furthermore, PSMs expression and staphylococcal enterotoxins (SEs) levels in relation to antibiotic sensitive and resistant strains were analysed. While the co-expression of PSMs and SEs was confirmed, one typical foodborne strain simultaneously yielding PSMs, SEB and SED was selected. By comparing this wildtype strain to a series of gene-deficient mutants, we concluded that PSMs and SEs expressions both relied on staphylococcal accessory regulator A initiation in the early stage of accessory gene regulator control, yet their succedent regulations differentiated to RNAlll-dependent and independent, respectively. These data provided preliminary insight into PSMs and SEs expression in foodborne S. aureus, and may guide the further studies on PSMs effects in SFP.
\end{abstract}

Keywords: Staphylococcus aureus, Phenol-soluble modulin, Staphylococcal enterotoxin, Foodborne, Co-expression

\section{Introduction}

Staphylococcus aureus (S. aureus) is not only a common cause of clinical infection, but also one of the leading foodborne pathogen worldwide (Kadariya et al. 2014). During recent decades, persistent antibiotic exposure has produced various resistant strains, among which Methicillin-resistant $S$. aureus (MRSA) is among the most notorious. In the 1990s, the emergence of Communityassociated MRSA (CA-MRSA) indicated that MRSA has spread out of the hospital and threatened the public health (Argudín and Mendoza 2010). The morbid $S$. aureus strains, multidrug-resistant variants in particular,

\footnotetext{
*Correspondence: 382951112@qq.com

${ }^{+} X$ Xiaoxiao Wu and Miao Yang contributed equally to this work

${ }^{1}$ National Center of Supervision Inspection on Processed Food \& Food Additives Quality, Nanjing Institute of Product Quality Inspection, No. 3 Jialingjiang East Street, Nanjing 210019, China

Full list of author information is available at the end of the article
}

are versatile in causing diseases ranging from mild toxic reactions to fatal infections such as pyemia, endocarditis and pneumonia (Klevens et al. 2007; Otto 2012; Waters et al. 2011).

As a recently discovered staphylococcal cytolysin family, phenol-soluble modulins (PSMs) are a group of small amphipathic peptides with $\alpha$-helical structure. In $S$. aureus, at least 7 PSMs have been discovered: PSM $\alpha 1$ to PSM $\alpha 4$, PSM $\beta 1$ and PSM $\beta 2$, and the $S$. aureus $\delta$-toxin (Chatterjee and Otto 2013; Otto 2014; Peschel and Otto 2013; Wang et al. 2007). Psm genes, which are located on the core genome, generate the shorter (20-25 amino acids) $\alpha$-type and the longer ( 44 amino acids) $\beta$-type peptides through post-translational cleavage (Wang et al. 2007). Among the typical strains separated from clinic, PSMs production is more abundant in CA-MRSA than in Hospital-associated MRSA (HA-MRSA), and extremely high in high-virulence strains such as USA300 and 
USA400 (Otto 2014; Peschel and Otto 2013). Generally, the physiological effects of PSMs include host cell lysing, biofilm forming, pro-inflammation and antimicrobial effect (Chatterjee and Otto 2013; Peschel and Otto 2013; Wang et al. 2007; Cheung et al. 2012; Geiger et al. 2012; Joo et al. 2011; Somerville et al. 2003; Surewaard et al. 2012, 2013; Tsompanidou et al. 2013). These multifaceted properties indicate that PSMs represent a novel global virulence factor, and also a kind of essential molecule in organism colonization during the commensal lifestyle (Otto 2014; Peschel and Otto 2013).

In the process of staphylococcal food poisoning (SFP), the key toxins are staphylococcal enterotoxins (SEs), a family of thermostable and gastrointestinal protease-tolerant superantigen (Marrack and Kappler 1990). The SEs vary greatly among $S$. aureus strains, which are regulated by multiple and often overlapping pathways under the influence of environmental factors (Fisher et al. 2018). Once the food is contaminated with S. aureus, the organism will be allowed to grow to a high cell density under appropriate environment, triggering the production of SEs. After the ingestion, victims develop abdominal pain, intense diarrhea and vomiting (Marrack and Kappler 1990). Since 2002, CA-MRSA strains have been successively separated from SFP outbreaks, indicating that CA-MRSA is strongly correlated with SFP in addition to clinical infection (Jones et al. 2002; Zhang et al. 2013).

Since PSMs and most of SEs are controlled by Agr system (Zhang et al. 2013; Carnes et al. 2010; Boisset et al. 2007), their simultaneous production in the lateexponential phase infers a possible synergistic effect. Moreover, the surfactant-like properties of PSMs may accelerate microbial spread in food matrix (Tsompanidou et al. 2013), and the antimicrobial effect may benefit the colonization and multiplying (Joo et al. 2011). According to an "outside-in" signaling mechanism, staphylococcal superantigens progressively interact with host cells to stimulate the immune reaction, accompanied by cytolysins which facilitate the disease production (Stach et al. 2014). To date, there has been no report on PSMs expression in foodborne $S$. aureus and its relation to antibiotic resistance. Furthermore, the co-expression and regulation of PSMs and SEs in foodborne strains have not yet been verified. Here, in order to initiate a research area into PSMs effect in food safety, we collected a panel of $S$. aureus isolates from various food, and assessed their PSMs production by a high-resolution mass spectrometry. Then, the correlation between PSMs and SEs expression, as well as the possible regulation pathways, were assayed in wild type and gene-deficient strains.

\section{Materials and methods}

\section{S. aureus strains}

A panel of foodborne $S$. aureus isolates was collected by the laboratories of the National Center of Food Testing \& Supervision and Jiangsu Provincial Center for Disease Control and Prevention. In addition, a number of S. aureus strains were derived from local hospital as the control. Twenty standard S. aureus strains, including USA300 and USA400, were also included (Additional file 1: Table S1). All strains were spread on Bradparker Agar (Luqiao, China), and then confirmed to be plasma-coagulase positive. Morphologically typical clones were isolated and incubated in Tryptic Soybean broth (Luqiao, China) at $37^{\circ} \mathrm{C}$ for subsequent studies.

\section{Antibiotic resistance assay}

Susceptibilities of $S$. aureus stains were tested using the Sensititre Susceptibility Plates for Clinical NonFastidious Organism PRCM1F (ThermoFisher, USA). One 96-well plate contained 7 serially diluted antibiotics coated on the wells: Oxacillin, Erythromycin, Tetracycline, Clindamycin, Ciprofloxacin, Vancomycin and Chloramphenicol. The assay was conducted according to the manufacturer's instructions. MIC values were determined by the terminal well of precipitation growth, and the susceptibilities were determined in accordance with NCCLS criteria.

\section{Detection of PSMs expression}

After overnight growth, S. aureus cells were separated by centrifugation. The culture supernatants were incubated at a $1: 1$ ratio with 1 -butanol at $37^{\circ} \mathrm{C}$ for $2 \mathrm{~h}$. The mixture was then centrifuged and the layer containing 1-butanol was collected and dried. Ultrapure water was used to redissolve the dried sample, which was immediately filtered through a syringe filter before LC-MS analysis.

A high performance liquid chromatography-quadrupole time-of-flight mass spectrometry (HPLC-QTOF) system (Agilent, USA) was used to analyze PSMs levels in the treated supernatant qualitatively and quantitatively. The chromatographic separation was performed with a Zorbax $300 \AA$ SB-C8 column $(3.5 \mu \mathrm{m}, 2.1 \times 150$ $\mathrm{mm}$ ) in series with a flow rate of $0.5 \mathrm{~mL} / \mathrm{min}$ and a binary solvent system with mobile phase A $(0.1 \%$ formic acid in water) and mobile phase B ( $0.1 \%$ formic acid in acetonitrile). The ESI experiments were carried out by Dual AJS ESI ion source. Signals acquired in MS mode were used to make a preliminary identification, and data obtained in targeted MS/MS mode were searched in the online Mascot database (http://www.matrixscience.com) to confirm 
the peptide sequence. As standard substances, all PSM peptides were synthesized by commercial vendors at $>95 \%$ purity with an $\mathrm{N}$-terminal $\mathrm{N}$-formyl methionine modification, as found in naturally occurring PSMs.

\section{SEs levels}

The presence of SEs in the S. aureus culture supernatants was firstly detected using the mini-Vidas Staphylococcal Enterotoxin II Kit (Bio Mérieux, France). SE-positive strains were selected and the expressions of SEA to SEE were quantified using $S$. aureus Enterotoxin $\mathrm{ABCDE}$ ELISA Kit (Ridascreen, Germany). Based on the ELISA principle, the concentrations of each SEs were determined by the standard curves derived from a series of standard samples.

\section{Construction of gene-deficient strains}

Different mutants derived from a typical foodborne $S$. aureus strain were constructed, in which the whole $a g r$ gene, RNAIII gene, agrA gene, sarA gene and rot gene were deleted, respectively. First, the upstream and downstream fragments (400 bp each) of each target gene were amplified, and then the recombinant genes in which the upstream and downstream fragments flanked the erythromycin $(\mathrm{Em})$ resistance gene were constructed. The recombinant genes were inserted into the shuttle vector pBT2 for allele replacement. The plasmids and primers used in this study are shown in Additional file 1: Table S2.

The S. aureus strain RN4220 at early-exponential phase was suspended in $0.5 \mathrm{M}$ sucrose, and kept on ice to yield electro-competent cells. The cells were transferred to a Gene Pulser cuvette, and the electro-transfer was performed on an ECM830 electroporator (BTX, USA). The electric settings were as follows: Voltage, $2.5 \mathrm{kV}$; capacitor, $50 \mu \mathrm{F}$; resistance, $200 \Omega$. After electroporation, the cells were immediately transferred into $400 \mu \mathrm{L}$ of TSB for $1 \mathrm{~h}$, placed on Em-containing medium and incubated overnight. Subsequently, the plasmid was extracted and electro-transformed into the target strain using the same procedure. The clones were incubated in B-medium at $30{ }^{\circ} \mathrm{C}$ for $12 \mathrm{~h}$ and left in $40{ }^{\circ} \mathrm{C}$ to grow overnight. The 1:100 diluted culture was inoculated into fresh B-medium, and $1 \mu \mathrm{L}$ of overnight culture was spread onto the agar plate until Em-resistant, Chloramphenicol $(\mathrm{Cm})$-sensitive clones were found. The mutations were confirmed by PCR amplification with specific primers.

\section{Statistical analysis}

The PSMs levels between S. aureus groups were compared using the Student's t test and One-way ANOVA. Linear analysis was applied to determine the correlation between PSMs and SEs co-expression. All statistics were performed using InStat 3.06 Software (Graphpad, La Jolla, CA, USA).

\section{Accession numbers}

All sequences of agr, RNAIII, agrA, sarA and rot were retrieved from the whole genome sequence of $S$. aureus strain USA300 (Accession No. AASB02000001.1).

\section{Results \\ PSMs expression in foodborne $S$. aureus}

A high resolution HPLC-ESI-QTOF approach was established to assay PSMs expressed by $S$. aureus strains. In positive ionization mode, the $[\mathrm{M}+\mathrm{nH}]^{\mathrm{n}+}$ ion peaks of 7 target PSMs were detected according to their accurate $\mathrm{m} / \mathrm{z}$ ratios, charges and retention time (Table 1 ). Further, data obtained in MS/MS were substituted into Mascot Software to confirm the peptide. The query results were in accordance with the published sequences and modification. Meanwhile, the representative mass spectrum of 7 PSMs in the supernatant were also identical to those of the synthesized peptides. The QTOF MS spectrum of PSMs in the mixed standard solution, as well as the extract ion chromatography (EIC) of 7 PSMs in USA300 are shown in Fig. 1. Taken the PSMs level of USA300 as $100 \%$, PSMs expressed by 3 different operons (PSM $\alpha 1-$ $4, \delta$ toxin and PSM $31-2$ ) in all strains were normalized (Additional file 1: Table S3). As shown in Fig. 2, the

Table 1 Amino acid sequence, $\mathrm{m} / \mathrm{z}$ ratios and retention times of $\mathrm{S}$. aureus PSMs

\begin{tabular}{|c|c|c|c|c|}
\hline \multirow{2}{*}{$\begin{array}{l}\text { PSMs } \\
\text { PSMa1 }\end{array}$} & \multirow{2}{*}{$\begin{array}{l}\text { Amino acid sequence } \\
\text { fMGIIAGIIKVIKSLIEQFTGK }\end{array}$} & \multicolumn{2}{|c|}{$\mathrm{MS}(\mathrm{m} / \mathrm{z} \text { ratios, } \mathrm{Da})^{\mathrm{a}}$} & \multirow{2}{*}{$\begin{array}{l}\text { RT (min) } \\
12.073\end{array}$} \\
\hline & & $763.4554(\mathrm{t})$ & $1144.6842(d)$ & \\
\hline PSMa2 & fMGIIAGIIKFIKGLIEKFTGK & $769.4640(t)$ & $1153.6964(d)$ & 11.639 \\
\hline PSMa3 & fMEFVAKLFKFFKDLLGKFLGNN & $879.1432(d)$ & $1318.2228(d)$ & 10.207 \\
\hline PSMa4 & fMAIVGTIIKIIKAIIDIFAK & $734.1283(\mathrm{t})$ & $1100.6982(d)$ & 20.819 \\
\hline PSM $\delta$-toxin & fMAQDIISTIGDLVKWIIDTVNKFTKK & $1002.8844(\mathrm{t})$ & $1503.8245(d)$ & 12.056 \\
\hline PSM 1 & fMEGLFNAIKDTVTAAINNDGAKLGTSIVSIVENGVGLLGKLFGF & $1131.8610(q)$ & $1508.8015(t)$ & 15.588 \\
\hline PSMB2 & fMTGLAEAIANTVQAAQQHDSVKLGTSIVDIVANGVGLLGKLFGF & $1121.8514(q)$ & $1495.4609(\mathrm{t})$ & 8.190 \\
\hline
\end{tabular}

${ }^{a}$ (d), (t) and (q) respectively stand for doubly, triply and quadruply charged ions 


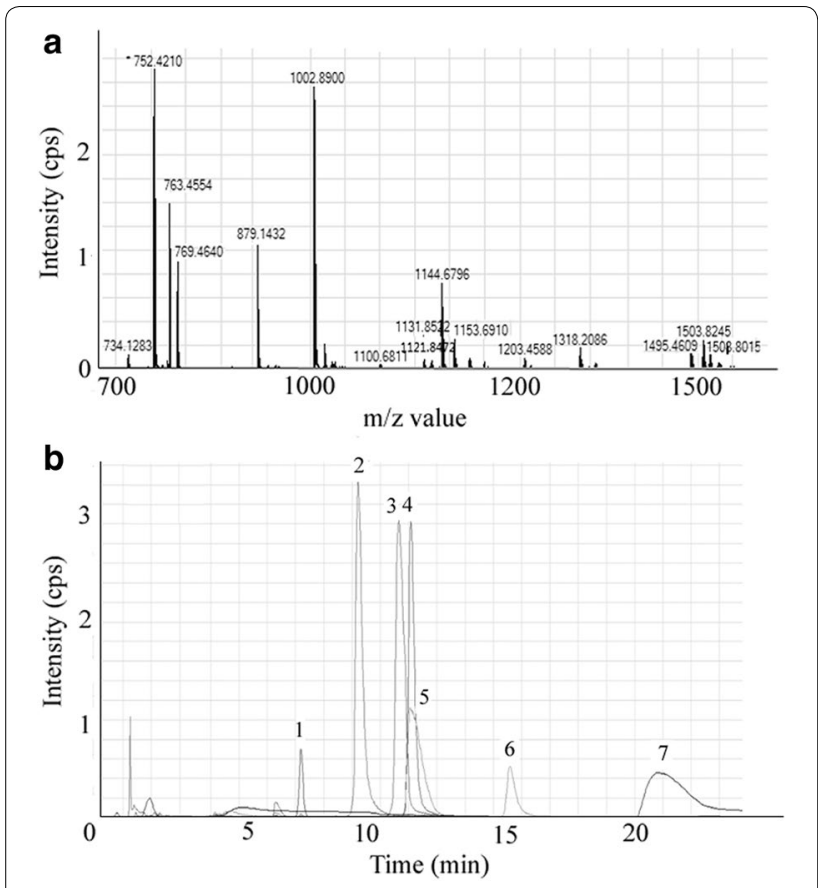

Fig. 1 QTOF MS spectrum and ion chromatograms of PSMs. a QTOF MS spectrum of PSMs in the mixed standard solution. $\mathbf{b}$ Extracted ion chromatograms of PSMs in USA 300 culture supernatant: 1, PSM 2 (m/z 1495.4609); 2, PSMa3 (m/z 879.1432); 3, PSMa2 (m/z 769.4640);

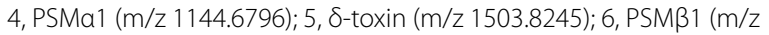
1508.8015); 7, PSMa4 (m/z 734.1283)

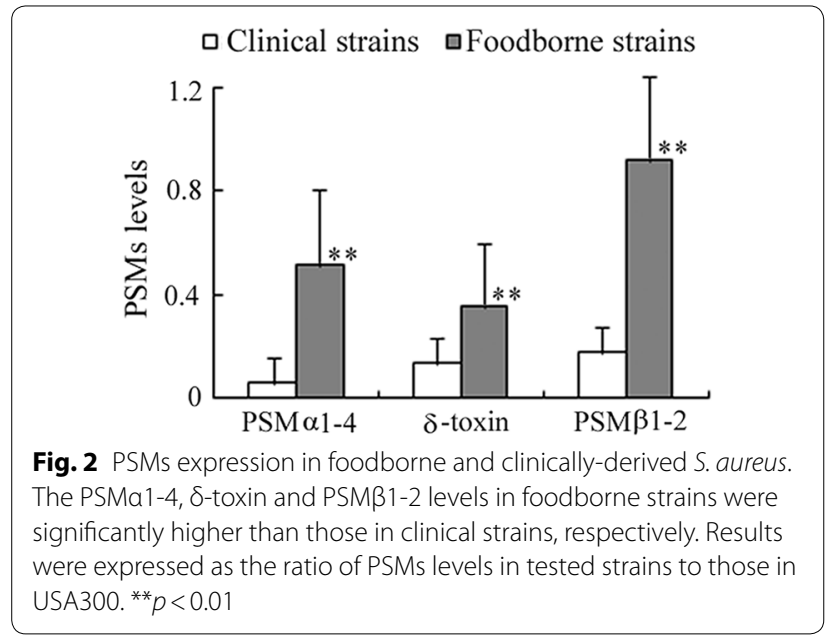

average PSMs levels of 350 food-derived strains were significantly higher than those of 127 isolates separated from patients $(p<0.01)$. In addition, among the standard $S$. aureus, only the CA-MRSA strains USA300 and USA400 exhibited extremely high PSMs production. In contrast, PSMs in most of standard HA-MRSA and methicilin susceptible Staphylococcus aureus (MSSA) strains were lower than $20 \%$ of those in USA300.

\section{PSMs levels in drug-resistant strains}

PSMs expression in common drug-resistant foodborne strains was analyzed. In all food-derived S. aureus, $77.4 \%$ (271/350) exhibited antibiotic resistance, among which 216 strains were multidrug resistant. The single or multiple antibiotic-resistant strains both exhibited markedly lower PSMs levels than the sensitive counterparts (Fig. 3). In the drug-resistance $S$. aureus, MRSA and vancomycin intermediate Staphylococcus aureus (VISA) strains produced mildly higher PSMs compared with other isolates, but these differences did not reach statistical significance (data not shown).

\section{Co-expression of PSMs and SEs in foodborne S. aureus}

SEA to SEE production in the culture supernatant of foodborne $S$. aureus was detected. The differences of PSMs levels between SE-positive and negative strains were not significant (data not shown). Yet, high correlations between SEs and PSMs productions were observed in the SE-positive S. aureus, indicating co-expression of PSMs and SEs. Table 2 lists the correlation coefficients and statistics between each SEs and PSMs.

\section{Regulation of PSMs and SEs co-expression}

A representative foodborne $S$. aureus strain, which simultaneously produced PSMs, SEB and SED in high levels, was selected for further study. A series of regulatory gene deficient strains derived from ZJY55 were constructed by homologous recombination, generating $\mathrm{agr}^{-1-}, \mathrm{RNAIII}^{-1-}, \mathrm{agrA}^{-1-}, \mathrm{rot}^{-1-}$ and $\mathrm{sarA}^{-1-}$ strains. The expressions of PSMs and SEs in wildtype and the derived strains were detected, and the possible mechanisms of gene regulation were summarized. As shown in Fig. 4, the expressions of PSMs, SEB and SED were diminished in the $a g r^{-/-}$and $a g r A^{-/-}$mutants, whereas, the RNAIII $^{-/-}$strain exhibited mildly decreased PSMs but sharply reduced SEs. In the rot gene deficient strain, the SEs levels significantly increased while the PSMs levels remained steady. Moreover, PSMs and SEs were both markedly reduced in the $s a r A$ deficient isolate.

\section{Discussion}

It is generally recognized that highly pathogenic CAMRSA strains produce higher amount of PSMs than HA-MRSA and MSSA, suggesting a tight correlation between PSMs and virulence (Otto 2014). To date, most studies on PSMs expression were conducted using typical strains separated from patients, including CA-MRSA, HA-MRSA and MSSA (Queck et al. 2009). PSM $\alpha$ links 
$\square$ Sensitive $\square$ Single resistant $\square$ Multiple resistant

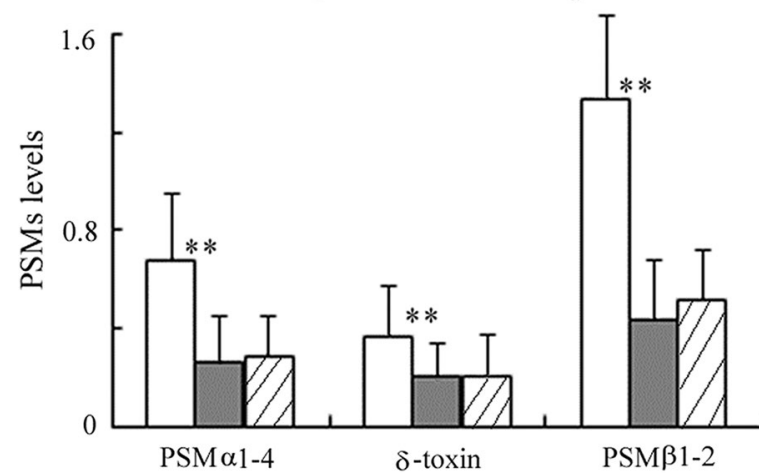

Fig. 3 PSMs expression in sensitive and drug-resistant foodborne S. aureus. The PSMs in single and multiple antibiotic resistant strains were significantly lower than those in sensitive strains, respectively. Results were expressed as the ratio of PSMs levels in tested strains to those in USA300. ${ }^{* *} p<0.01$

Table 2 Correlation coefficients between SEs and PSMs levels in foodborne $S$. aureus

\begin{tabular}{llllll}
\hline $\begin{array}{l}\text { Correlation } \\
\text { coefficients }\end{array}$ & SEA & SEB & SEC & SED & SEE \\
\hline PSMa & $0.4065^{*}$ & $0.5344^{* *}$ & $0.5430^{* *}$ & $0.6006^{* *}$ & $0.4120^{*}$ \\
$\delta$ toxin & 0.3552 & $0.6214^{* *}$ & $0.4115^{*}$ & $0.6522^{* *}$ & 0.3260 \\
PSM $\beta$ & $0.5251^{*}$ & $0.5501^{* *}$ & $0.5334^{* *}$ & $0.5365^{* *}$ & $0.4661^{*}$ \\
\hline
\end{tabular}

${ }^{*} p<0.05,{ }^{* *} p<0.01$

the key roles of cell lysis, and also contributes to biofilm forming (Otto 2014; Peschel and Otto 2013). In addition, PSMs contribute to phenotypes not associated with infection, thereby representing the original mechanisms for bacteria survival, out of which the function of virulence factors evolved (Otto 2014; Periasamy et al. 2012). Food-derived S. aureus, usually separated due to the positive reaction of plasma-coagulase, was deemed to show dual capability for food colonization and pathogenicity. During food contamination, a serious of surface proteins, cytolins and superantigens are successively expressed, facilitating the $S$. aureus dissemination and colonization. In the foodborne $S$. aureus, expressions of PSMs and its correlations with drug resistance have not been reported. Here, we collected $S$. aureus strains from various foods to detect the PSMs levels, as well as the pattern of their antibiotic resistance. The foodborne $S$. aureus exhibited relatively higher PSMs expressions in contrast to the clinical isolates, inferring a marked significance of PSMs in the lifecycle of foodborne isolates. Generally, MRSA with multiple antibiotic resistances exhibited relatively high capability in host invasion and existence. In some strains, the emerging of anti-drug resistance was accompanied with the lost of certain virulence genes. When bacteria accepted the plasmid containing resistance genes, the levels of toxic factors might shift (Soto 2009). Here, we revealed that antibiotic-resistant foodborne $S$. aureus synthesized a lower PSMs levels than the sensitive isolates. It indicated that PSMs could be involved in the "original" virulence of $S$. aureus in promoting colonization or emulsifying nutrients (Peschel and Otto 2013), and might be impaired during the acquirement of the antibiotic-resistant properties to adapt the environment.

$S$. aureus secretes a wide variety of virulence factors which are strictly controlled by a complex regulatory network (Kadariya et al. 2014; Delgado et al. 2008). PSMs and most SEs are strictly controlled by Agr quorum sensing, yet their mechanisms are distinct. SEB and SED are both widely distributed superantigens in SFP which are regulated by the ordinary Agr pathway (Novick 2003). In contrast, the Agr control of PSMs is RNAIII-independent. PSMs expression is triggered by direct binding of agr $A$ to the psm promoter regions, which may coupled with additional regulation other than by agr (Queck et al. 2009). In this study, we observed the co-expression between PSMs and SEs in foodborne S. aureus. Then, a
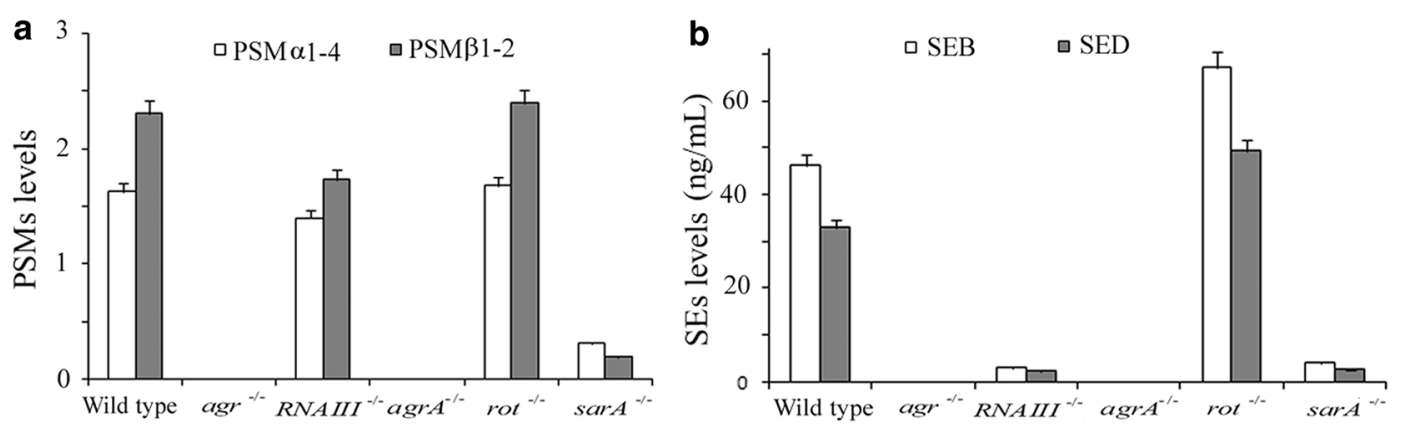

Fig. 4 PSMs and SEs expression in representative foodborne S. aureus and its variants. Results were Mean \pm SD of three experimental replications. a PSMs expression, expressed as the ratio of PSMs in tested strains to those in USA300. b SEs expression 
series of mutants of the typical isolate simultaneously producing PSMs, SEB and SED were used to investigate the regulation of gene expression. The whole Agr system was essential for the high production of both SEs and PSMs, and the co-expression hinges on sarA binding onto the agr promoter region to initiate transcription (Queck et al. 2008; Zielinska et al. 2011). Further, SEs production was under the regular control of RNAIII via removing rot inhibition, whereas, the expression of PSMs was only mildly downgraded by RNAIII, yet uninfluenced in the rot gene deleted strain. Accordingly, we concluded that during the early stage of Agr control, PSMs and SEs expressions both rely on the agr $A / B$ quorum sensing initiated by sarA. However, the subsequent regulations of SEs and PSMs were RNAIII-dependent and independent, respectively. So, additional regulatory elements may be involved in PSMs expression.

An LC-QTOF mass spectrum was employed for PSMs detection in the supernatants of $S$. aureus culture. This high-resolution methodology could accurately discriminate each target peptides from simply pre-treated samples, avoiding the disturbance of molecules with similar $\mathrm{m} / z$ values. In addition, the output file of $\mathrm{ms} / \mathrm{ms}$ mode could be searched in the database of protein mass spectrum to confirm the primary structure and modifications. In a previous investigation, according to the imprecise $m / z$ detected by HPLC system coupled with an Ion Trap mass spectrometer, the $m / z 1046.1$ peak was initially suggested to be an ADM2 fragment, but subsequently proven to be derived from PSM $\alpha$ after antimicrobial analysis (Joo et al. 2011). Here, the LC-QTOF method confirmed the precise $m / z$, charges, experimental condition and procedures, thus categorically averted the erroneous assessment for target peptides. Furthermore, this approach is preferable to assay peptides in complicated substrates such as food and environmental samples due to its high resolution. We also detected PSMs in artificial contaminated milk and meat using this method, and found massive interfering peptides with similar $\mathrm{m} / z$ to PSMs, which were impossible be differentiated by former MSD Trap SL mass spectrometer.

Recently, an "outside-in" signaling model has been illustrated by Stach et al. (2014), describing a possible staphylococcal virulence mechanism via the combination of superantigens and cytolins. In this model, the pathogenic superantigen binds to epithelial cells by the aid of a certain cytolysin, and thereby triggers the cascade of preinflammatory signals to disrupt the permeability barrier. Then, the produced cytokines/chemokines attract cells of innate and adaptive immune system to induce massive inflammation and facilitate the disease production (Chatterjee and Otto 2013). However, no combination of superantigen and cytolin has been verified to perform a synergistic reaction in SFP through "outside-in signaling". The statistical data of PSMs and SEs' correlation and co-expression raised the possibility that they may act as mutual promotional elements in food poisoning. Further evidence on cell lines and animal models are now indicated to investigate the pathogenic role of PSMs and SEs.

\section{Additional file}

Additional file 1: Table S1. Standard S. aureus strains. Table S2. Plasmids and primers used in this study. Table S3. Determination of PSMs in standard S. aureus strains.

\section{Abbreviations}

PSMs: staphylococcal phenol-soluble modulins; SFP: staphylococcal food poisoning; SEs: staphylococcal enterotoxins; SarA: staphylococcal accessory regulator A; Agr: accessory gene regulator; MRSA: Methicillin-resistant S. aureus; CA-MRSA: Community-associated MRSA; HA-MRSA: Hospital-associated MRSA

\section{Authors' contributions}

CZ designed the research and supported the project. XW and MY performed the major experiments, analyzed data and wrote the paper. XF carrying out additional experiments and analysis. The remaining authors contributed to refining the ideas and revised the manuscript. All authors read and approved the final manuscript.

\section{Author details}

${ }^{1}$ National Center of Supervision Inspection on Processed Food \& Food Additives Quality, Nanjing Institute of Product Quality Inspection, No. 3 Jialingjiang East Street, Nanjing 210019, China. ${ }^{2}$ Key Laboratory of Child Development and Learning Science (Ministry of Education), School of Biological Science \& Medical Engineering, Southeast University, No. 2 Sipailou Street, Nanjing 210018, China. ${ }^{3}$ Jiangsu Provincial Center for Disease Control and Prevention, No. 172 Jiangsu Street, Nanjing 210009, China. ${ }^{4}$ Beijing Entry-Exit Inspection and Quarantine Bureau Inspection and Quarantine Technical Center, No. 6 Tianshuiyuan Street, Chaoyang District, Beijing 100026, China.

\section{Acknowledgements}

We would like to express the great gratitude to Professor Sun Baolin in University of Science and Technology of China for providing the plasmids.

\section{Competing interests}

The authors declare that they have no competing interests.

Availability of data and materials

Not applicable.

\section{Consent for publication}

Not applicable.

Ethics approval and consent to participate

This article does not contain any studies with human participants or animals performed by any of the authors.

\section{Funding}

This work was supported by National Key R\&D Program of China (2018YFF0215200 and 2017YFF0211201), Science \& Technology Project of Jiangsu Quality and Technical Supervision Bureau (KJ185613), Science \& Technology Project of China General Administration of Quality Supervision, Inspection and Quarantine (2015QK055), Science and Technology Program of Nanjing, China (201716005). 


\section{Publisher's Note}

Springer Nature remains neutral with regard to jurisdictional claims in published maps and institutional affiliations.

Received: 18 July 2018 Accepted: 17 November 2018

Published online: 22 November 2018

\section{References}

Argudín MA, Mendoza MC, Rodicio MR (2010) Food poisoning and Staphylococcus aureus enterotoxins. Toxins 2:1751-1773

Boisset S, Geissmann T, Huntzinger E, Fechter P, Bendridi N, Possedko M, Chevalier C, Helfer AC, Benito Y, Jacquier A, Gaspin C, Vandenesch F, Romby P (2007) Staphylococcus aureus RNAIll coordinately represses the synthesis of virulence factors and the transcription regulator rot by an antisense mechanism. Genes Dev 21:1353-1366

Carnes EC, Lopez DM, Donegan NP, Cheung A, Gresham H, Timmins GS, Brinker CJ (2010) Confinement-inducedquorumsensing of individual Staphylococcus aureus bacteria. Nat Chem Biol 6:41-45

Chatterjee SS, Otto M (2013) How can Staphylococcus aureus phenol-soluble modulins be targeted to inhibit infection? Future Microbiol 8:693-696

Cheung GY, Duong AC, Otto M (2012) Direct and synergistic hemolysis caused by Staphylococcus phenol-soluble modulins: implications for diagnosis and pathogenesis. Microbes Infect 14:380-386

Delgado A, Zaman S, Muthaiyan A, Nagarajan V, Elasri MO, Wilkinson BJ, Gustafson JE (2008) The fusidic acid stimulon of Staphylococcus aureus. J Antimicrob Chemoth 62:1207-1214

Fisher EL, Otto M, Cheung GYC (2018) Basis of virulence in enterotoxin-mediated staphylococcal food poisoning. Front Microbiol 9:436

Geiger T, Francois P, Liebeke M, Fraunholz M, Goerke C, Krismer B, Schrenzel J, Lalk M, Wolz C (2012) The stringent response of Staphylococcus aureus and its impact on survival after phagocytosis through the induction of intracellular PSMs expression. PLoS Pathog 8:e1003016

Jones TF, Kellum ME, Porter SS, Bell M, Schaffner W (2002) An outbreak of community-acquired foodborne illness caused by methicillin-resistant Staphylococcus aureus. Emerg Infect Dis 8:82-84

Joo HS, Cheung GY, Otto M (2011) Antimicrobial activity of community-associated methicillin-resistant Staphylococcus aureus is caused by phenolsoluble modulin derivatives. J Biol Chem 286:8933-8940

Kadariya J, Smith TC, Thapaliya D (2014) Staphylococcus aureus and staphylococcal food-borne disease: an ongoing challenge in public health. Biomed Res Int 2014:827965

Klevens RM, Morrison MA, Nadle J, Petit S, Gershman K, Ray S, Harrison LH, Lynfield R, Dumyati G, Townes JM, Craig AS, Zell ER, Fosheim GE, McDougal LK, Carey RB, Fridkin SK, Active Bacterial Core surveillance (ABCs) MRSA Investigators (2007) Invasive methicillin-resistant Staphylococcus aureus infections in the United States. JAMA 298:1763-1771

Marrack P, Kappler J (1990) The staphylococcal enterotoxins and their relatives. Science 248:705-711
Novick RP (2003) Autoinduction and signal transduction in the regulation of staphylococcal virulence. Mol Microbiol 48:1429-1449

Otto M (2012) MRSA virulence and spread. Cell Microbiol 14:1513-1521

Otto M (2014) Phenol-soluble modulins. Int J Med Microbiol 304:164-169

Periasamy S, Chatterjee SS, Cheung GY, Otto M (2012) Phenol-soluble modulins in Staphylococci: what are they originally for? Commun Integr Biol 5:275-277

Peschel A, Otto M (2013) Phenol-soluble modulins and staphylococcal infection. Nat Rev Microbiol 11:667-673

Queck SY, Jameson-Lee M, Villaruz AE, Bach TH, Khan BA, Sturdevant DE, Ricklefs SM, Li M, Otto M (2008) RNAlll-independent target gene control by the agr quorum-sensing system: insight into the evolution of virulence regulation in Staphylococcus aureus. Mol Cell 32:150-158

Queck SY, Khan BA, Wang R, Bach TH, Kretschmer D, Chen L, Kreiswirth BN, Peschel A, Deleo FR, Otto M (2009) Mobile genetic element-encoded cytolysin connects virulence to methicillin resistance in MRSA. PLoS Pathog 5:e1000533

Somerville GA, Cockayne A, Dürr M, Peschel A, Otto M, Musser JM (2003) Synthesis and deformylation of Staphylococcus aureus $\delta$-toxin are linked to tricarboxylic acid cycle activity. J Bacteriol 185:6686-6694

Soto SM (2009) Relationship between virulence and antimicrobial resistance in bacteria. Rev Med Microbiol 20(4):84-90

Stach CS, Herrera A, Schlievert PM (2014) Staphylococcal superantigens interact with multiple host receptors to cause serious diseases. Immunol Res 59:177-181

Surewaard BG, Nijland R, Spaan AN, Kruijtzer JA, de Haas CJ, van Strijp JA (2012) Inactivation of staphylococcal phenol soluble modulins by serum lipoprotein particles. PLoS Pathog 8:e1002606

Surewaard BG, de Haas CJ, Vervoort F, Rigby KM, DeLeo FR, Otto M, van Strijp JA, Nijland R (2013) Staphylococcal alpha-phenol soluble modulins contribute to neutrophil lysis after phagocytosis. Cell Microbiol 15:1427-1437

Tsompanidou E, Denham EL, Becher D, de Jong A, Buist G, van Oosten M, Manson WL, Back JW, van Dij JM, Dreisbach A (2013) Distinct roles of phenol-soluble modulins in spreading of Staphylococcus aureus on wet surfaces. Appl Environ Microbiol 79:886-895

Wang R, Braughton KR, Kretschmer D, Bach TH, Queck SY, Li M, Kennedy AD, Dorward DW, Klebanoff SJ, Peschel A, DeLeo FR, Otto M (2007) Identification of novel cytolytic peptides as key virulence determinants for community-associated MRSA. Nat Med 13:1510-1514

Waters AE, Contente-Cuomo T, Buchhagen J, Liu CM, Watson L, Pearce K Foster JT, Bowers J, Driebe EM, Engelthaler DM, Keim PS, Price LB (2011) Multidrug-resistant Staphylococcus aureus in US meat and poultry. Clin Infect Dis 52:1227-1230

Zhang C, Shen Y, Dong MS (2013) Distribution, polymorphism and temporal expression of egc in Staphylococcus aureus isolates from various foods in China. Food Control 29:279-285

Zielinska AK, Beenken KE, Joo HS, Mrak LN, Griffin LM, Luong TT, Lee CY, Otto M, Shaw LN, Smeltzer MS (2011) Defining the strain-dependent impact of the Staphylococcal accessory regulator (sarA) on the a-toxin phenotype of Staphylococcus aureus. J Bacteriol 193:2948-2958

\section{Submit your manuscript to a SpringerOpen ${ }^{\odot}$ journal and benefit from:}

- Convenient online submission

- Rigorous peer review

- Open access: articles freely available online

- High visibility within the field

- Retaining the copyright to your article

Submit your next manuscript at $\boldsymbol{\nabla}$ springeropen.com 